\title{
VIDEO
}

\section{New methods of teaching the axis in ERCP: Can I write my name?}
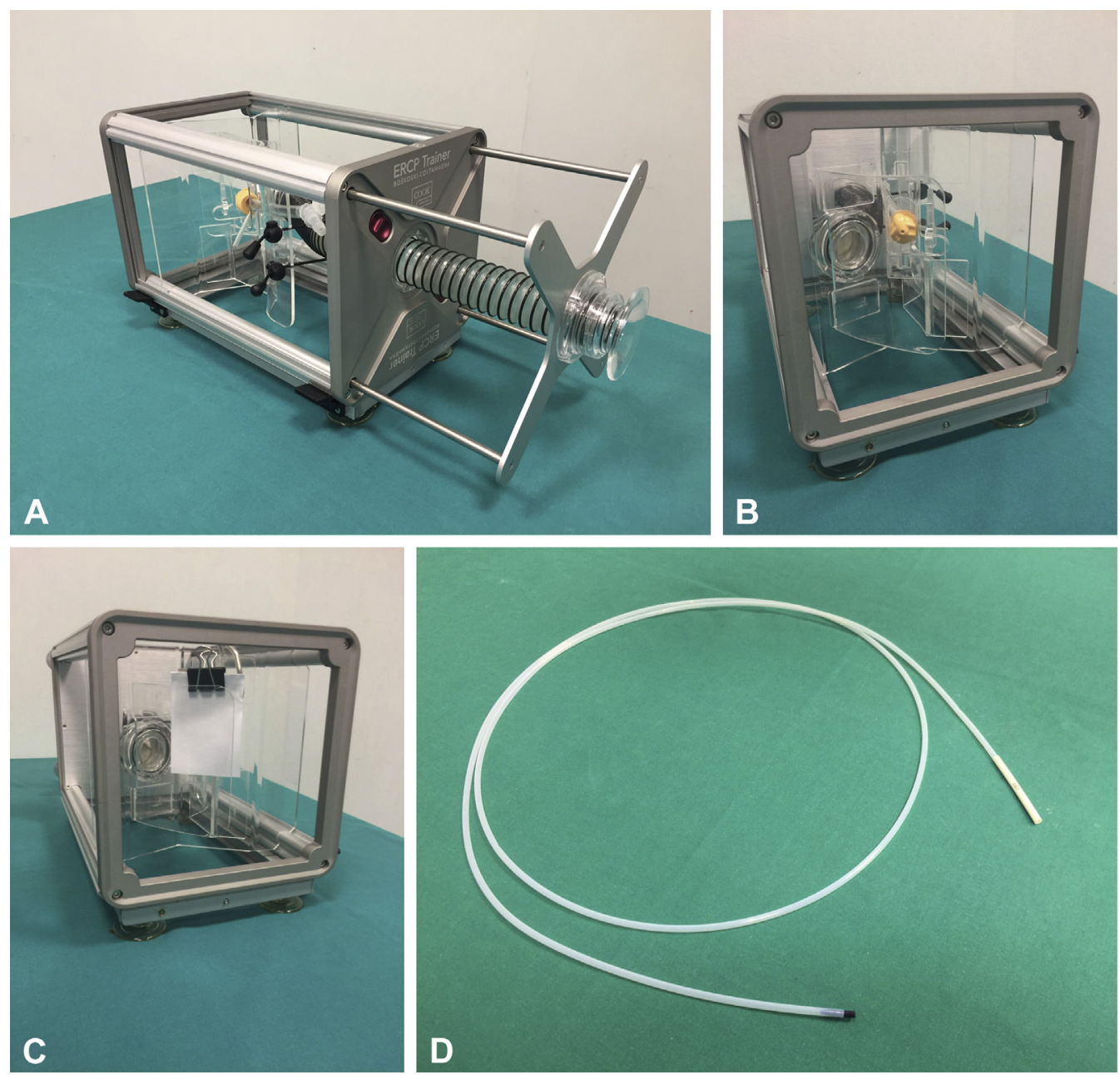

Figure 1. A, The Boškoski-Costamagna ERCP Trainer. B, C, The papilla before $(\mathbf{B})$ and after $(\mathbf{C})$ placement of the paper for writing. D, The handmade ERCP writing accessory made of a $10 \mathrm{~F}$ stent pusher and a pen mounted on the top.

ERCP is among the most difficult procedures in gastrointestinal endoscopy. In fact, as testified by P. B. Cotton in the 1960s to the 1970s, ERCP was "difficult to overstate," and it still is today. Learning ERCP demands patience, skills, and an understanding of cholangiopancreatographic anatomy and imaging interpretation, as well as the ability to gain the cannulation axis and choose the adequate accessories. Most of all, knowing how to deal with ERCP-related adverse events is probably the key to "standing alone."

ERCP training in patients can be very risky. Simulators allow the practicing of invasive endoscopic procedures in a controlled environment with no risk to patients. Four types of simulators are available today: live animals (pigs and dogs), explanted organs, mechanical devices, and computer simulators. The Boškoski-Costamagna ERCP Trainer (Cook Medical, Limerick, Ireland) is a newly designed mechanical simulator that allows practicing all maneuvers in ERCP (eg, basic and advanced cannulation, stone extraction, stent placement) with the simulated patient in the prone, supine, and oblique positions (Video 1 , available online at www.VideoGIE.org). The biggest problem for trainees in ERCP is gaining the correct axis 
in front of the papilla, including bad coordination of the wheels, the elevator, and the shaft, together with the fear of doing something wrong. To overcome all of this, and to show to young trainees that any axis in front of the papilla can be reached in any direction, we conducted an experiment. For this purpose, we used the BoškoskiCostamagna ERCP Trainer (Fig. 1A). The papilla was removed and replaced by a piece of paper (Figs. 1B and $\mathrm{C}$ ). A handmade ERCP writing accessory was made of a $10 \mathrm{~F}$ stent pusher (Cook Medical) with a pen mounted on its top (Fig. 1D). Using coordinated circular and straight movements of the big and small wheels and the shaft of the endoscope, and coordinated movements of the elevator, we easily managed to write the word "ERCP."

This new type of training can be reproduced in any circumstance with little cost and in any endoscopy unit in the world where ERCP is practiced. We believe that this new "out of the box" approach will help trainees in fearless learning of this complex procedure.

\section{DISCLOSURE}

Drs Boškoski and Costamagna are consultants for Cook Medical. All other authors disclosed no financial relationships relevant to this publication.

Ivo Boškoski, MD, PhD, Fondazione Policlinico Gemelli, Rome, Italy, IHU, Istitut de Chirurgie Guidée par I'Image, Strasbourg, France, Rosario Landi, MD, Vincenzo Bove, MD, Catholic University of Rome, Rome, Italy, Andrea Tringali, MD, PhD, Fondazione Policlinico Gemelli, Rome, Italy, Catholic University of Rome, Rome, Italy, Guido Costamagna, MD, FACG, Fondazione Policlinico Gemelli, Rome, Italy, Catholic University of Rome, Rome, Italy, IHU, USIAS Strasbourg University, Strasbourg, France

Copyright (c) 2018 American Society for Gastrointestinal Endoscopy. Published by Elsevier Inc. This is an open access article under the CC BY-NC-ND license (http://creativecommons.org/licenses/by-nc-nd/4.0/).

https://doi.org/10.1016/j.vgie.2017.10.008 